\section{Mit onkolytischen Viren gegen Leberkrebs}

Bei Patienten mit fortgeschrittenem Leberkrebs, bei denen Sorafenib nicht angeschlagen hat, wird nach neuen Optionen gesucht. In einer US-amerikanisch-koreanischen Studie wurde nun das Potenzial onkolytischer Viren bei Patienten im Stadium N1M1 ausgelotet.

$\mathrm{n}$ einer Phase-I-Studie wurde mit dem onkolytischen Virus JX-594 der US-Firma Jennerex bei soliden Tumoren ein komplettes Ansprechen sowie eine systemische Wirkung erzielt. Nun prüften südkoreanische und US-Onkologen die Wirksamkeit der intratumoralen Injektion des gentechnisch veränderten Vaccinia-Virus in niedriger und hoher Dosis.

Zunächst waren 49 Patienten mit Leberzellkrebs behandelt worden. Bereits früh zeichnete $a b$, dass der Nutzen im Hinblick auf das Überleben in hoher Dosierung größer war, sodass die Aufnahme von Patienten gestoppt wurde.

Für eine ausreichende statistische Aussagekraft reichten schließlich insgesamt 30 Patienten, 16 mit hoher und 14 mit niedriger intratumoral injizierter Virusdosis. Die meisten Tumoren waren im BCLC (Barcelona Clinic Liver Cancer)-Stadium C, also Stadium N1M1 plus Gefäßinvasion.

Das verwendete Virus war genetisch mit dem Gen für den Wachstumsfaktor GM-CSF sowie für das Enzym $\beta$-Galaktosidase ausgestattet. Der Wachstumsfaktor dient der Immunstimulation, das Enzym als Marker für die Vermehrung des Virus. Dreimal wurde die Virussuspension im Abstand von je zwei Wochen injiziert.

Insgesamt wurde die Therapie gut vertragen. Bereits 15 Minuten nach Injektion tauchte das Virus in höchster Konzentration im Blut auf. Das Ansprechen auf die Therapie war in beiden Dosierungsgruppen gleich groß, die Überlebenszeit bei Patienten mit hoher Dosierung mit median 14,1 Monaten signifi- kant höher als in der Vergleichsgruppe mit 6,7 Monaten (Hazard Ratio $0,39 ; \mathrm{p}=0,020)$. In einer aktuellen Phase-III-Studie mit Sorafenib waren es zwischen zwei und vier Monaten gewesen.

Nach einem Jahr lebten noch $66 \%$ der Behandelten in der Hochdosis-, in der Vergleichsgruppe $23 \%$. Die Überlebensrate nach eineinhalb Jahren betrug 35 vs. $11 \%$. Die 19 Patienten mit mehreren Tumorherden überlebten median nur halb so lange wie die zehn Patienten mit nur einem Herd in der Leber (8,8 vs. 16,6 Monate).

Fazit: Bei Patienten mit fortgeschrittenem Leberkrebs, bei denen Sorafenib nicht anschlägt, kann die Behandlung mit einem onkolytischen Vaccinia-Virus die Lebenszeit deutlich verlängern. Der Effekt soll nun gegen Placebo überprüft werden.

Peter Leiner

Heo J et al. Randomized dose-finding clinical trial of oncolytic immunotherapeutic vaccinia JX-594 in liver cancer. Nat Med. 2013;19(3):32936.

\title{
HCC: RAF-Kinasen effektiv hemmen
}

Die Inhibition onkogener Kinasen stellt derzeit die einzige Therapieoption mit nachgewiesener lebensverlängernder Wirkung beim fortgeschrittenen primären Leberzellkarzinom (HCC) dar. Der Aktivierungsstatus des EGFR scheint eine Schlüsselrolle in der Resistenzentwicklung zu spielen.

$\mathrm{D}$ er klinische Effekt einer Behandlung mit dem Multikinaseinhibitor Sorafenib beschränkt sich bei Patienten mit primärem HCC häufig auf eine temporäre Tumorstabilisierung. Mangels valider prädiktiver Marker stellt die Identifizierung von Patienten mit voraussichtlich gutem Ansprechen auf diese Therapie eine große Herausforderung dar. Sorafenib wurde ursprünglich als Inhibitor der RAF-Kinasen ARAF, BRAF und CRAF/Raf-1 identifiziert, denen eine wichtige Rolle in der Transduktion zellproliferierender Signale zukommt. Neben der RAS/RAF/MEK/ERK-Kaskade als wichtigem Target geraten auch verschiedene Rezeptortyrosinkinasen wie VEGFR ins therapeutische Visier.

Welche Mechanismen dem unterschiedlichen Ansprechen von HCC-Zellen auf Sorafenib und der Resistenzentwicklung zugrundeliegen, untersuchten die Autoren in vitro an sechs genetisch differierenden Hepatom-Zelllinien. Dabei offenbarten sich dosisabhängig deutliche Unterschiede im Ansprechen und bei der Inhibition von RAF/MEK/ERKund PKB-mTOR-Kaskade.

Weiteres wichtiges Ergebnis: Resistente Zellkulturen zeichneten sich durch hohe EGFR-Aktivität aus, umgekehrt steigert eine EGFR-Blockade die Sorafenib-Sensitivität. Angesichts der offenbar moderaten Wirksamkeit von EGFR-Inhibitoren als Monosubstanzen legen die Ergebnisse einen synergistischen Effekt der Kombination mit Sorafenib, mutmaßlich durch eine verbesserte Blockade der RAF-Kinasen, nahe.

Fazit: EGFR-Aktivierung scheint eine wichtige Rolle in der primären Resistenzentwicklung von HCC-Zelllinien gegenüber Sorafenib zu spielen. Diese Ergebnisse bergen das Potenzial für eine weitere Therapieoptimierung und könnten die Identifizierung prädiktiver Biomarker in diesem Kontext voranbringen.

Wolfgang Zimmermann

Ezzoukhry Z et al. EGFR activation is a potential derminant of primary resistance of hepatocellular carcinoma cells to sorafenib. Int J Cancer. 2012;131(12):2961-9. 